\title{
Effects of prenatal multimicronutrient supplementation on pregnancy outcomes: a meta-analysis
}

\author{
Prakesh S. Shah MD MSc, Arne Ohlsson MD MSc, on behalf of the Knowledge Synthesis Group \\ on Determinants of Low Birth Weight and Preterm Births
}

An abridged version of this article appeared in the June 9, 2009, issue of $C M A J$ and is available at www.cmaj.ca/cgi/content/full/cmaj.081777/DC1.

\section{ABSTRACT}

Background: Reduced intake of micronutrients during pregnancy exposes women to nutritional deficiencies and may affect fetal growth. We conducted a systematic review to examine the efficacy of prenatal supplementation with multimicronutrients on pregnancy outcomes.

Methods: We searched MEDLINE, EMBASE, CINAHL and the Cochrane Library for relevant articles published in English up to December 2008. We also searched the bibliographies of selected articles as well as clinical trial registries. The primary outcome was low birth weight; secondary outcomes were preterm birth, small-for-gestational-age infants, birth weight and gestational age.

Results: We observed a significant reduction in the risk of low birth weight among infants born to women who received multimicronutrients during pregnancy compared with placebo (relative risk [RR] $0.81,95 \%$ confidence interval [Cl] 0.73-0.91) or iron-folic acid supplementation (RR $0.83,95 \% \mathrm{Cl} 0.74-0.93)$. Birth weight was significantly higher among infants whose mothers were in the multimicronutrient group than among those whose mothers received iron-folic acid supplementation (weighted mean difference $54 \mathrm{~g}, 95 \% \mathrm{Cl} 36 \mathrm{~g}-72 \mathrm{~g}$ ). There was no significant differences in the risk of preterm birth or small-forgestational-age infants between the 3 study groups.

Interpretation: Prenatal multimicronutrient supplementation was associated with a significantly reduced risk of low birth weight and with improved birth weight when compared with iron-folic acid supplementation. There was no significant effect of multimicronutrient supplementation on the risk of preterm birth or small-for-gestational-age infants.

Une version française de ce résumé est disponible à l'adresse www.cmaj.ca/cgi/content/full/cmaj.081777/DC2

CMAJ 2009;180(12):E99-E108

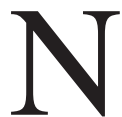
utrition plays an important role in the growth and development of the fetus. Studies of the nutritional status of pregnant women during the Dutch famine revealed increased risks of infertility, abortion, fetal intrauterine growth restriction and perinatal mortality among malnourished women. ${ }^{1}$ In many parts of the world, a similar situation exists for many pregnant women with respect to nutrition. Overall, the diet of pregnant women has been reported to be deficient in calories and micronutrients. ${ }^{2}$ Both macro- and micronutrients are important for a woman to sustain pregnancy and for appropriate growth of the fetus.

The exact mechanisms of how supplementation with micronutrients can affect pregnancy outcomes are not completely understood. Possible mechanisms for beneficial effects include a generalized improvement in the immune function of women, with a reduced incidence of infections and subsequent reduced incidence of preterm birth ${ }^{3}$ improved energy metabolism and anabolic processes in the mother, with a reduced incidence of fetal intrauterine growth restriction; ${ }^{3}$ improved ability to respond to stress; ${ }^{3}$ expansion of plasma volume secondary to fluid retention, with subsequent improvements in fetal growth $;{ }^{4}$ improved hemoglobin levels, ${ }^{5}$ and increased absorption of iron related to intake of vitamin $\mathrm{C}$ and riboflavin, with subsequent improvement in hemoglobin levels.

Potential disadvantages include adverse interactions of micronutrients when supplied in combination; ${ }^{6}$ enhanced or reduced absorption of one nutrient by other nutrients (e.g., interaction between iron and vitamin $\mathrm{C}$, and iron and zinc); ${ }^{7}$ deleterious effects on the fetus and the mother from overdose of any one component (e.g., vitamin A overdose) ${ }^{6}{ }^{6}$ and cost. ${ }^{6}$

Potential barriers include the lack of well-defined government policies on maternal health and nutrition. ${ }^{6}$ A multicomponent approach has been criticized from the standpoint that some micronutrients may be necessary, some may not be needed and some may even be harmful. ${ }^{2}$ Generalized or mass supplementation with multimicronutrients may have different effects on pregnancy outcomes depending on the underlying nutritional status of the women.

On the basis of a systematic review performed in 2005, the World Health Organization currently recommends iron-folic acid supplementation for all pregnant women. ${ }^{8.9}$ The review reported that multimicronutrient supplementation during pregnancy were more efficacious than 2 or fewer micronutrients in

From the Department of Paediatrics (Shah, Ohlsson), Mount Sinai Hospital and University of Toronto, and the Departments of Health Policy, Management and Evaluation (Shah, Ohlsson), and Obstetrics and Gynaecology (Ohlsson), University of Toronto, Toronto, Ont. 
reducing the rates of low birth weight and small-forgestational-age births. However, when multimicronutrients were compared with iron-folic acid supplementation, no evidence of a difference was noted. ${ }^{7}$ Further research in this area was encouraged because information was derived from a few reports. Since then, several randomized controlled trials have evaluated the efficacy of multimicronutrients and have reported varied results. With advancement in our knowledge from recently reported trials, ${ }^{10}$ we conducted a systematic review and meta-analysis of the efficacy of supplementation with multimicronutrients during pregnancy in reducing the rates of low birth weight, preterm birth and small-for-gestational-age births compared with placebo or iron-folic acid supplementation.

\section{Methods}

We conducted this systemic review to examine whether supplementation with multimicronutrients during pregnancy reduces the risk of low birth weight, preterm birth and small-forgestational-age births compared with placebo and compared with iron-folic acid supplementation.

\section{Literature search}

We searched MEDLINE, EMBASE, CINAHL and the Cochrane Library (from their inception to Dec. 15, 2008) for all relevant studies without language restriction. The search terms used for MEDLINE are reported in Appendix 1 (available at www.cmaj.ca/cgi/content/full/cmaj.081777/DC3); they were modified according to database requirements. We reviewed the bibliographies of the identified articles to locate further eligible studies. In addition, we searched 20 clinical trial registries (Appendix 2, available at www.cmaj.ca/cgi /content/full/cmaj.081777/DC3), abstracts of the last 2 years from conferences of the Society of Maternal and Fetal Medicine and the Society of Obstetricians and Gynaecologists of Canada, and Google Scholar ${ }^{\mathrm{TM}}$ for ongoing or completed trials.

\section{Study selection and validity assessment}

We included randomized and quasi-randomized trials (randomization based on possible identifiers such as date of birth, hospital number or day of the week) that explored the efficacy of supplementation with multimicronutrients during pregnancy and reported any of the outcomes of interest. We included only information available from publications and did not contact primary authors for additional data.

We included studies in which women received multimicronutrient supplementation at any time during pregnancy. A previous systematic review ${ }^{7}$ had excluded studies of patients who were HIV positive because of concerns over their background nutritional status. To improve the generalizability of our findings, we included all populations. We planned sensitivity analyses with data for all patients and with data for HIV-positive women.

We included studies that compared multimicronutrient supplementation with placebo or with iron-folic acid supplementation. We did not restrict the selection of trials on the basis of the specific combination or number of micronutrients. We anticipated that most studies would have used the recommended dietary allowance of individual micronutrients. We planned sub- group analyses to assess the effects of studies in which supplementation of most of the micronutrients were in doses higher or lower than the recommended dietary allowance. Studies of supplementation that started at any time during pregnancy and for any length of time were included. We planned a subgroup analysis to assess the effect of when supplementation was started (before or after 20 weeks' gestation). We report on compliance with supplementation as described by the authors of the studies.

We excluded studies in which prepared food containing macro- and micronutrients was given rather than a unique supplement of micronutrients. We also excluded studies in which the outcomes were not clearly defined. We reviewed studies published only as abstracts and included them only if quality assessment could be completed from the given information. Studies for which quality could not be determined were excluded. We excluded letters, commentaries, reviews and editorials if they did not contain original data.

One of us (P.S.S.) scanned the titles and abstracts of the articles initially. Selected articles were retrieved in full; both of us (P.S.S. and A.O.) assessed them for eligibility. We resolved discrepancies by consensus.

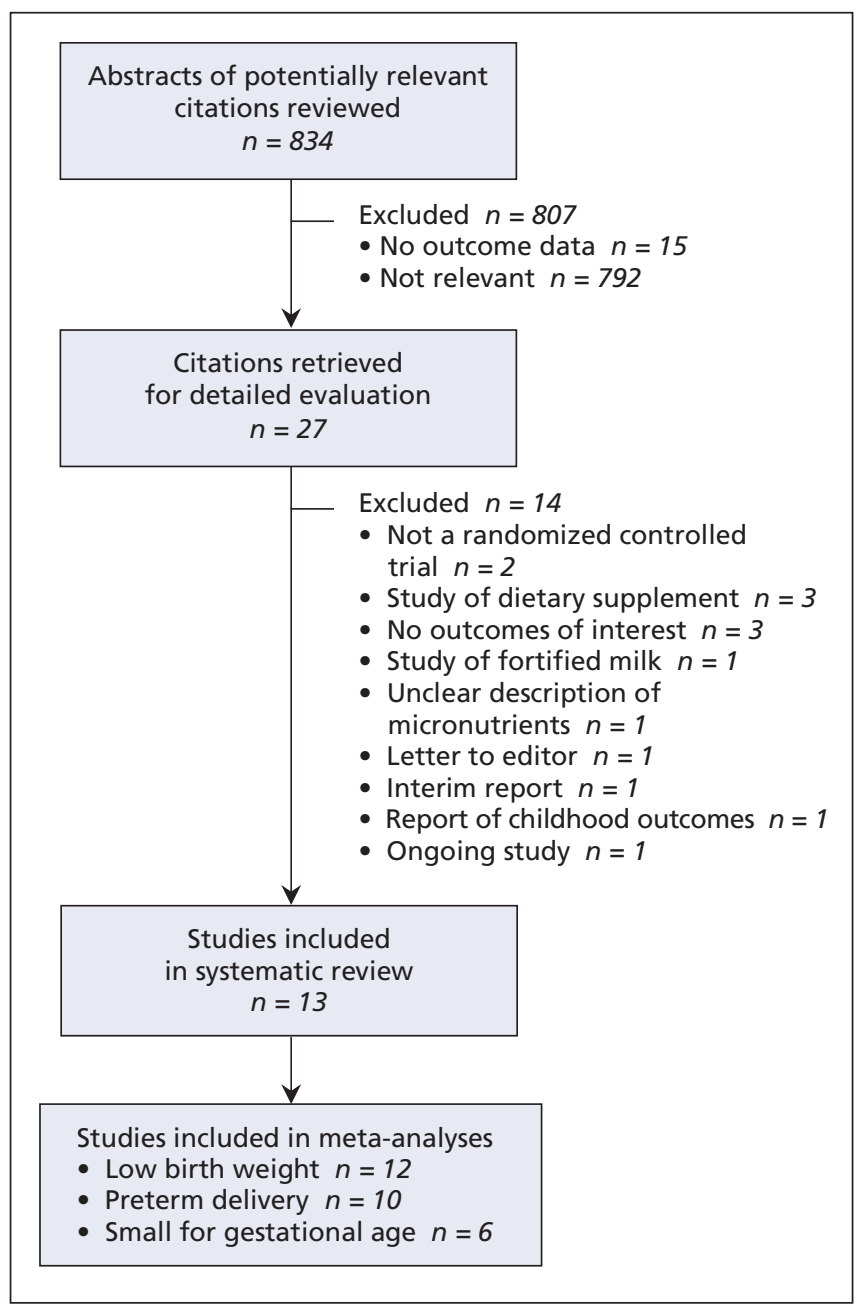

Figure 1: Selection of studies for meta-analysis of the effect of prenatal supplementation with multimicronutrients on pregnancy outcomes. 


\section{Outcome measures}

The primary outcome of interest was the incidence of low birth weight $(<2500 \mathrm{~g})$. Secondary outcomes were preterm birth (birth before 37 weeks' gestation), small-for-gestationalage infants $(<10$ th centile for gestational age or $>2$ standard deviations below the mean for gestational age), birth weight (in grams) and gestational age (in weeks).

\section{Methodologic quality}

Both of us (P.S.S. and A.O.) independently assessed the methodologic quality of the studies using a predefined checklist, as suggested for the Cochrane Database of Systematic Reviews. ${ }^{11}$ This checklist assesses risk of bias in the categories of sequence generation, allocation concealment, blinding, attrition, selective reporting and other biases (Appendix
3, available at www.cmaj.ca/cgi/content/full/cmaj.081777 /DC3). The classification in each category was yes, no, and cannot tell or unclear. We performed an overall assessment of risk of bias, based on responses from the above-mentioned criteria. We resolved discrepancies by consensus. We planned sensitivity analyses that would exclude studies with a high likelihood of bias in 3 or more domains.

We assessed statistical heterogeneity using the Cochran Q test and by calculating $I^{2}$ values. Clinical heterogeneity was assessed for timing of supplementation, methodologic quality of the studies and characteristics of the study populations. We assessed publication bias using the funnel plot method.

\section{Data extraction and synthesis}

Two of us (P.S.S. and A.O.) extracted data from each eligible

Table 1: Brief description of the studies included in the meta-analysis of multimicronutrient supplementation during pregnancy

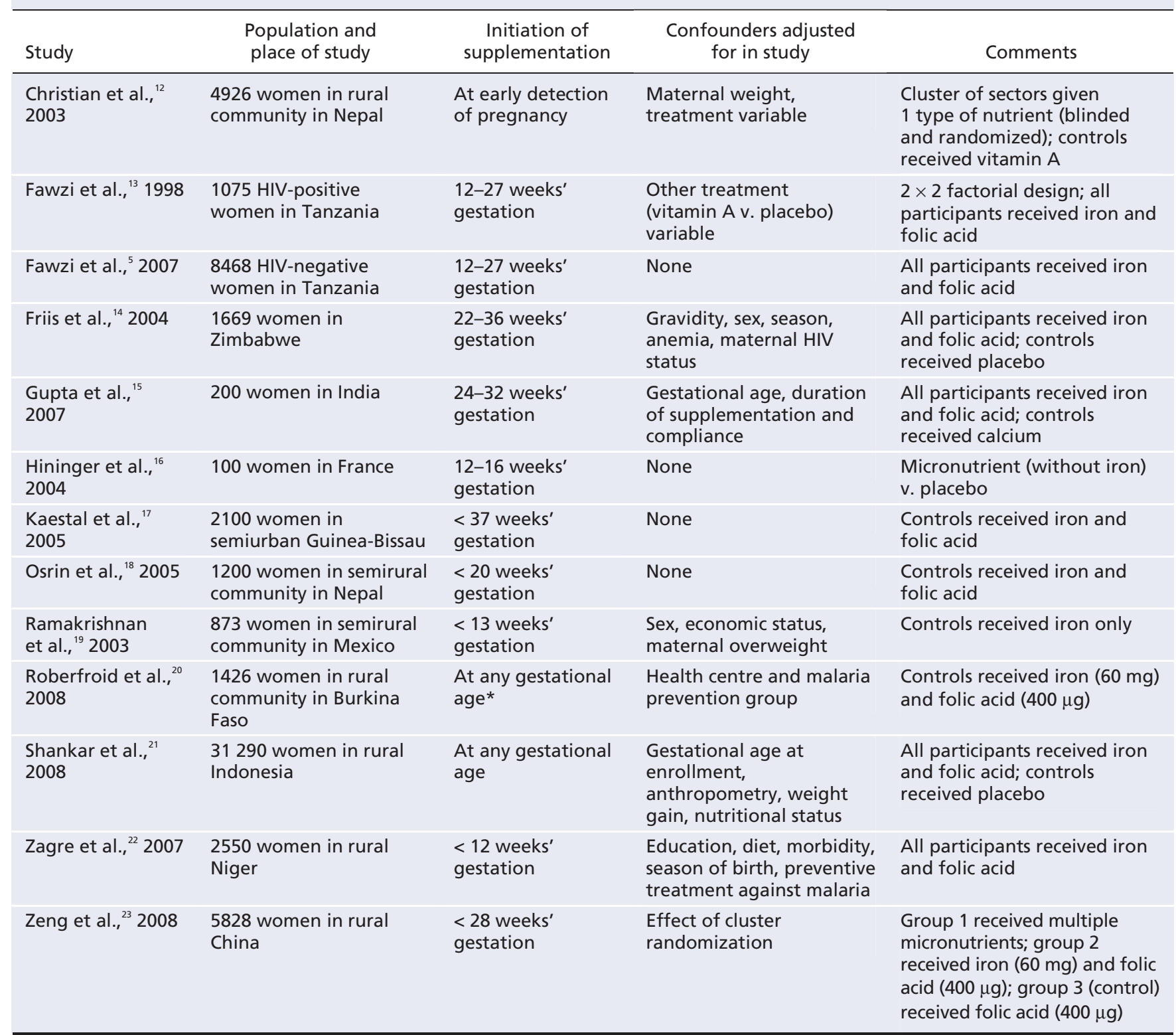


study using data-collection forms. We resolved discrepancies by consensus. The original data were not modified. At times, calculations were required from available data for the meta-analysis.

For binary outcome variables (low birth weight, preterm birth and small for gestational age), we calculated the relative risks (RRs), risk differences and numbers needed to benefit and to harm, as well as respective $95 \%$ confidence intervals (CIs). For continuous measures (birth weight and gestational age), we calculated the mean differences or standardized mean differences as appropriate. Weighting of the studies in the meta-analyses was calculated based on the inverse variance of the study. We expected clinical and statistical heterogeneity among the studies. Thus, we used the random-effect model for meta-analyses because it accounts for random variability both within and among studies. We planned sensitivity analyses to assess the effects of the quality of the studies on the results. No adjustment for multiple analyses was made.

\section{Results}

\section{Literature search}

We identified 13 trials ( 8 randomized trials, 1 factorial randomized trial and 4 cluster-randomized trials) of prenatal multimicronutrient supplementation for inclusion in the metaanalysis (Figure 1)..$^{5,2-23}$ We excluded 14 reports (the reasons for exclusion are given in Appendix 4, available at www.cmaj .ca/cgi/content/full/cmaj.081777/DC3).

\section{Study participants and interventions}

The baseline characteristics of the women in the included studies and the timing of initiation of micronutrient supplementation are reported in Table 1 . The composition of micronutrients in the different studies is reported in Table 2 and online Appendix 5 (available at www.cmaj.ca/cgi/content/full /cmaj.081777/DC3).

In the first report by Fawzi and colleagues, ${ }^{13}$ only HIVpositive patients were included, whereas in the report by Friis and colleagues ${ }^{14}$ approximately one-third of the patients were positive for HIV. We are aware that generalized undernutrition (protein, calories and micronutrients) could be a major issue in patients with HIV infection. However, we included these patients in the review to improve generalizability of the findings. In the second study by Fawzi and colleagues, ${ }^{5}$ the authors included only HIV-negative women and compared multivitamins with placebo. However, because all of the women received iron and folic acid, we included data for the control group in the iron-folic acid group.

Kaestel and colleagues ${ }^{17}$ studied 3 groups: a control group and 2 groups receiving micronutrients (one receiving about the equivalent of the recommended dietary allowance, and the other receiving about twice the recommended dietary allowance). For analyses purposes, we combined the data for the 2 micronutrient groups. We did not perform the sensitivity analyses of the effect of doses higher than the recommended dietary allowances because there was only 1 study.

Christian and colleagues ${ }^{12}$ included 5 groups: a control group and 4 groups receiving micronutrients (folic acid alone, folic acid and iron, folic acid and iron and zinc, or multimicronutrients). For the purpose of analysis, we compared the multimicronutrient group with the control group and with the group that received iron and folic acid.

Ramakrishnan and colleagues ${ }^{19}$ used iron only as supplementation in the control group. We included the data from this study in the placebo group.

Roberfroid and colleagues ${ }^{20}$ compared women receiving a micronutrient group to an iron-folic acid group.

Table 2: Composition of micronutrients in studies included in the meta-analysis

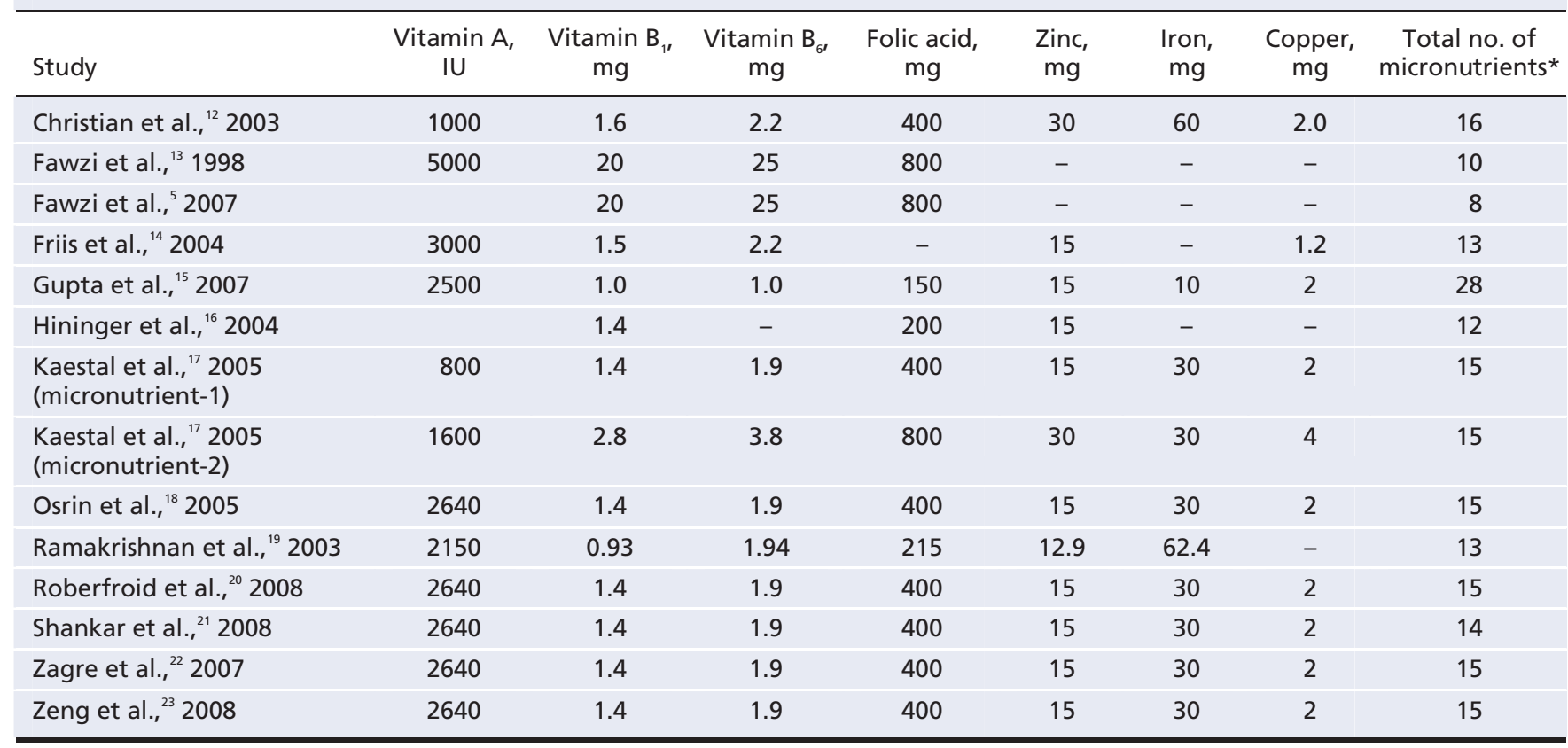

*A detailed description of other micronutrients in each study appears in Appendix 5 (available at www.cmaj.ca/cgi/content/full/cmaj.081777/DC3). 
Zeng and colleagues ${ }^{23}$ had 3 study groups: women who received multimicronutrients, those who received iron and folic acid and those who received folic acid alone (control group). For our analyses, we included data from the control group in the placebo group.

\section{Methodologic quality}

The results of our assessment of the risk of bias among included studies and compliance rates are reported in Appendix 6 (available at www.cmaj.ca/cgi/content/full/cmaj.081777 /DC3). All of the studies were of high quality. A few studies lost points for not providing enough details about sequence generation or allocation concealment.

\section{Outcomes}

Details of the outcomes reported in the individual studies are reported in Appendix 7 (available at www.cmaj.ca/cgi /content/full/cmaj.081777/DC3).

Twelve of the studies reported on the outcome of low birth weight (Figure 2). Four compared multimicronutrient supplementation with a placebo $(n=6097)$ and 10 compared it with iron-folic acid supplementation ( $n=29$ 889); 2 studies reported on both comparisons. The risk of low-birth-weight infants was significantly lower among women given multimicronutrients than among those given placebo (RR 0.81, 95\% CI 0.73 to 0.91 ; risk difference $-0.03,95 \% \mathrm{CI}-0.05$ to -0.01 ; number needed to benefit 33, 95\% CI 20 to 100). The same was true for the comparison with iron-folic acid supplementation (RR $0.83,95 \% \mathrm{CI}$ 0.74 to 0.93 ; risk difference $-0.02,95 \% \mathrm{CI}-0.03$ to -0.01 ; number needed to benefit $50,95 \%$ CI 33 to 100 ).

Ten studies reported on preterm birth as an outcome measure (Figure 3). Three studies compared multimicronutrient supplementation with placebo $(n=5191)$ and 9 compared it with iron-folic acid supplementation $(n=45192) ; 2$ studies reported on both comparisons. The risk of preterm birth was not significantly lower among women given multimicronutrient supplementation during pregnancy than among those given placebo (RR $0.97,95 \%$ CI 0.82 to 1.13 ) or iron-folic acid supplementation (RR $0.99,95 \%$ CI 0.96 to 1.03 ).

Six studies reported on the risk of small-for-gestational-age infants (Figure 4). Three studies compared multimicronutrient supplementation with placebo $(n=5140)$ and 5 compared it with iron-folic acid supplementation $(n=13039) ; 2$ studies reported on both comparisons. There was no statistically significant reduction in the risk of this outcome among women given multimicronutrient supplementation compared with women given placebo (RR $0.85,95 \%$ CI 0.71 to 1.02 ) or ironfolic acid supplementation (RR 0.89, 95\% CI 0.77 to 1.01 ).

The effect on birth weight was studied in 11 trials (Figure 5). Five studies compared multimicronutrient supplementation with placebo $(n=5727)$ and 8 compared it with iron-folic acid supplementation $(n=28033)$; 2 studies reported on both com-

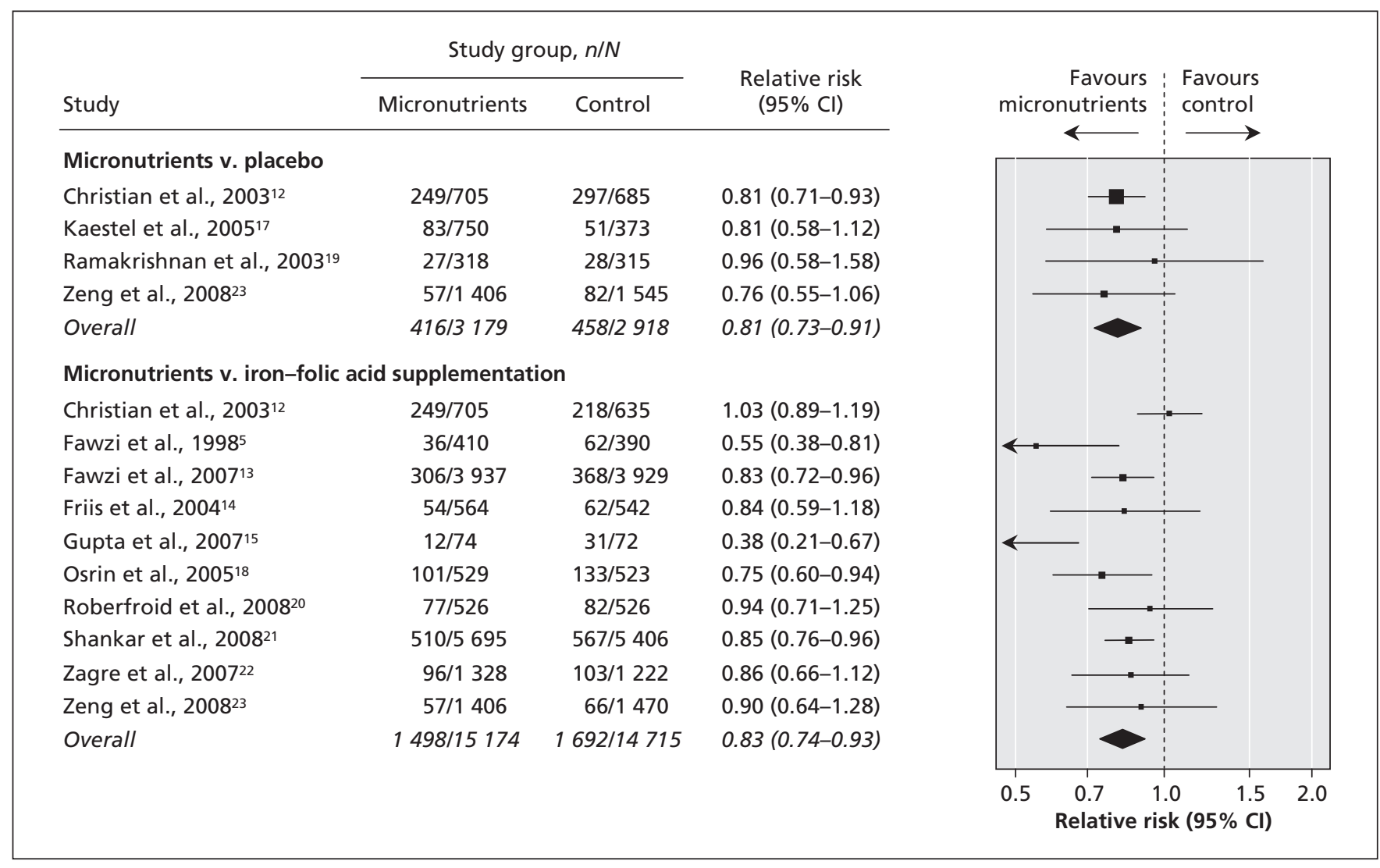

Figure 2: Random-effect meta-analysis of the risk of low-birth-weight infants among women given multimicronutrient supplementation versus control (placebo or iron-folic acid supplementation) during pregnancy. Values less than 1.0 indicate decreased risk of outcome with multimicronutrient supplementation. Note: $\mathrm{Cl}=$ confidence interval. 
parisons. There was no statistically significant increase in birth weight among infants born to women given multimicronutrient supplementation compared with women in the placebo arm (weighted mean difference $31 \mathrm{~g}, 95 \% \mathrm{CI}-5 \mathrm{~g}$ to $68 \mathrm{~g}$ ). Birth weight was significantly higher among infants born to women given micronutrient supplementation than among those whose mothers were given iron-folic acid supplementation (weighted mean difference $54 \mathrm{~g}, 95 \% \mathrm{CI} 36 \mathrm{~g}$ to $72 \mathrm{~g}$ ).

Of the 7 studies that reported the effect on gestational age, 3 compared multimicronutrient supplementation with placebo $(n=3867)$ and 5 compared it with iron-folic acid supplementation $(n=13523)$; 1 study reported on both comparisons (Figure 6). There was no significant difference in gestational age between infants of women given micronutrient supplementation and those of women given placebo (weighted mean difference 0.4 weeks, $95 \%$ CI -0.26 weeks to 0.34 weeks). The same was true for the comparison with iron-folic acid supplementation (weighted mean difference 0.00 weeks, $95 \%$ CI -0.10 weeks to 0.10 weeks).

\section{Heterogeneity and publication bias}

Clinical heterogeneity among the studies is described in Table 1. There was moderate statistical heterogeneity for low birth weight $\left(I^{2}=55 \%-64 \%\right)$ but not for preterm birth $\left(I^{2}=0 \%\right)$.

Visual inspection of the funnel plot suggested publication bias for the outcomes low birth weight and preterm birth for studies of small effect size showing both positive and negative results (Appendix 8, available at www.cmaj.ca/cgi/content/full
/cmaj.081777/DC3). We believe that this bias is unlikely to change the overall results, since the majority of included studies were of high quality and precision. However, this should be interpreted with caution in face of heterogeneity in studies. ${ }^{24}$

\section{Subgroup and sensitivity analyses}

In the end, we did not conduct a subgroup analysis of the effect of recommended dietary intake or more than adequate dietary intake through supplementation on pregnancy outcomes because only 1 study reported such data.

In a subgroup analysis based on the timing of initiation of supplementation, we found no difference in the risk of lowbirth-weight infants when multimicronutrient supplementation was compared with iron-folic acid supplementation (RR 0.89, 95\% CI 0.72 to 1.08 for initiation before 20 weeks' gestation; RR $0.80,95 \%$ CI 0.69 to 0.91 for initiation anytime after 12 weeks' gestation). However, the majority of the studies in the later category had entry criteria of 12-27 weeks' gestation.

We found that the effect of multimicronutrient supplementation was more pronounced for low-birth-weight and smallfor-gestational-age infants in randomized trials (low birth weight: RR $0.74,95 \%$ CI 0.62 to 0.89 ; small for gestational age: RR $0.82,95 \%$ CI 0.73 to 0.92 ) than in cluster-randomized trials (low birth weight: RR $0.91,95 \%$ CI 0.82 to 1.02 ; small for gestational age: RR $0.98,95 \%$ CI 0.84 to 1.14 ).

We performed a post-hoc stratified analysis to identify an effective minimal composition of micronutrients that can be suggested for practical use and to explore heterogeneity in the com-

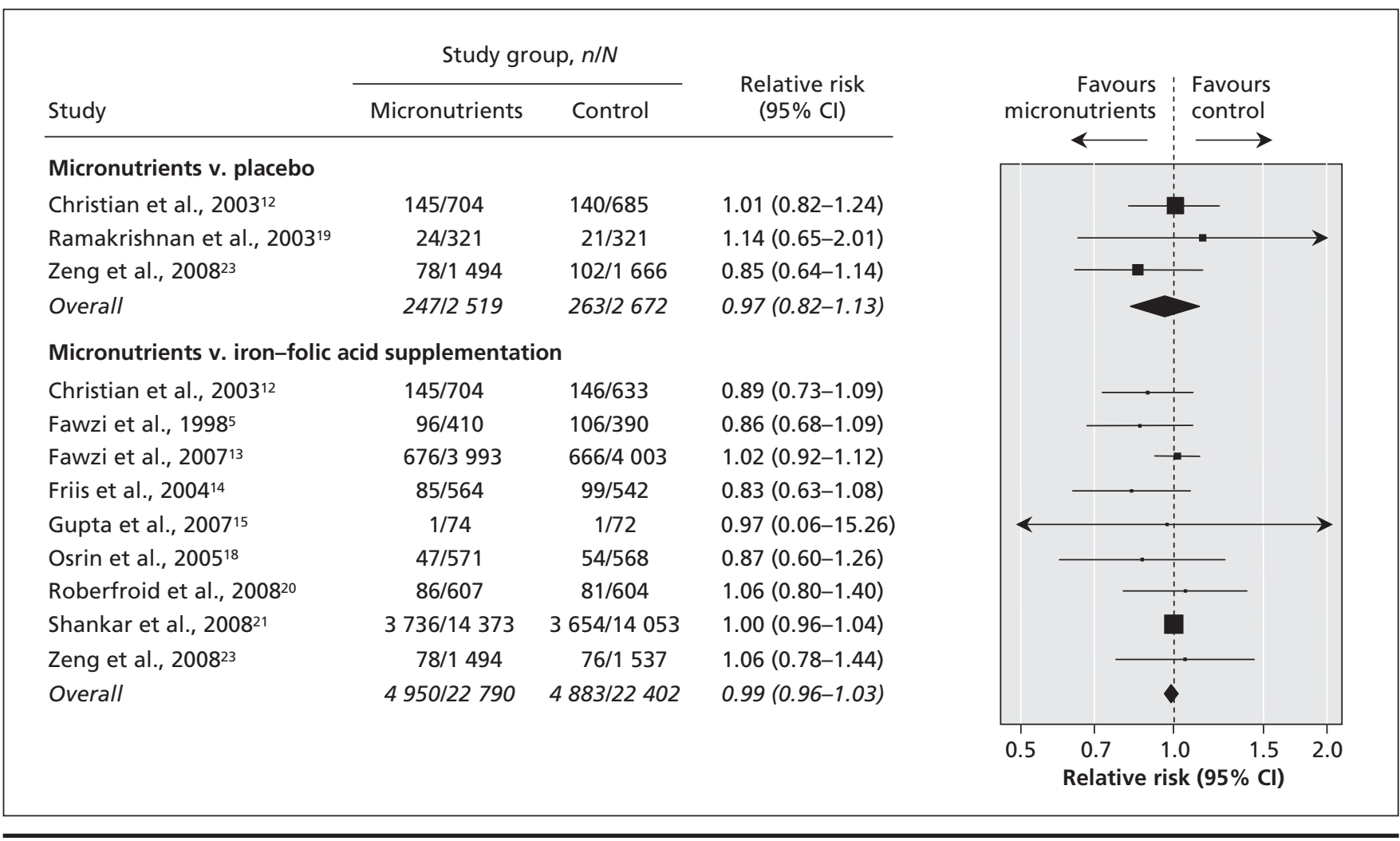

Figure 3: Random-effect meta-analysis of the risk of preterm birth among women given multimicronutrient supplementation versus control (placebo or iron-folic acid supplementation) during pregnancy. Values less than 1.0 indicate decreased risk of outcome with multimicronutrient supplementation. Note: $\mathrm{Cl}=$ confidence interval. 
position of micronutrients. We selected a group of studies that used a homogeneous combination of water and fat-soluble vitamins and minerals (vitamin $\mathrm{A} \geq 2640 \mathrm{IU}$, vitamin $\mathrm{D} \geq 200 \mathrm{IU}$, vitamin $E \geq 10 \mathrm{mg}$, vitamin $B_{1} \geq 1.4 \mathrm{mg}$, folic acid $\geq 400 \mu \mathrm{g}$, vitamin $C \geq 70 \mathrm{mg}$, zinc $\geq 15 \mathrm{mg}$ and iron $\geq 30 \mathrm{mg}$ ). We observed a reduction in the risk of low-birth-weight infants in this subgroup analysis (RR $0.85,95 \%$ CI 0.78 to $0.93, I^{2}=0 \%$ ). Despite the exclusion of studies that did not satisfy one or more criteria, we found no clear discernible pattern to indicate that one or more micronutrients in addition of iron and folic acid would suffice.

When we examined results for women with known HIV infection from 2 studies $(n=1160)$, we found no evidence of a difference in the risk of low-birth-weight infants between women given multimicronutrient supplementation and those given ironfolic acid supplementation (RR 0.73, 95\% CI 0.40 to 1.33 ).

\section{Interpretation}

In this systematic review, we identified a significantly reduced risk of low-birth-weight infants among women given multimicronutrient supplementation during pregnancy compared with women given placebo or iron-folic acid supplementation. The birth weight of infants was $54 \mathrm{~g}$ higher on average among those whose mothers were given micronutrients than among those whose mothers received iron-folic acid supplementation. We found no evidence of a significant difference in the risk of preterm birth or small-for-gestationalage infants between the study groups.

There was a certain degree of clinical and statistical heterogeneity among the studies included in the meta-analysis. This was not beyond what one could have predicted from the outset. The most important aspects of heterogeneity were the un- derlying population and the time of onset of supplementation.

The populations studied varied from undernourished women, adequately nourished women, HIV-positive women and confirmed HIV-negative women. In their first report, Fawzi and colleagues ${ }^{13}$ included only HIV-positive women, who may have been undernourished. We argue that this feature improves the generalizability of our findings. We realize that their inclusion may introduce bias toward a positive impact. However, if these or any other results are to be applied to the global population, where most women are unaware of their HIV status, it hardly justifies exclusion of a known HIVpositive population from these analyses. Our subgroup analysis of HIV-positive women did not reveal an improvement in the reduction of low-birth-weight infants, which supports our rationale of including such women in the combined analyses, since these individuals were not responsible for higher than expected contribution to the overall results. The patients included in most of these studies are truly representative of the global population. The studies were performed in resource-limited settings, exactly the place where efficacy of this intervention is warranted. The practice in most developed countries of giving multivitamins along with iron and folic acid to pregnant women ${ }^{5}$ needs to be adopted in developing countries. Similar to the approach taken in a previous Cochrane review, ${ }^{7}$ we included studies that reflected real-life scenarios but that we felt were homogeneous enough to be included in our meta-analysis.

The included trials were of very good quality. Although they all had missing data for birth outcomes for a proportion of patients, the results were unlikely affected because the numbers with missing data were small and were proportionally distributed. In some studies, the micronutrient supplementation was started at the earliest detection of pregnancy,

\begin{tabular}{|c|c|c|c|c|c|c|c|}
\hline \multirow[b]{2}{*}{ Study } & \multicolumn{2}{|c|}{ Study group, $n / N$} & \multirow{2}{*}{$\begin{array}{l}\text { Relative risk } \\
\quad(95 \% \mathrm{Cl})\end{array}$} & \multirow{2}{*}{\multicolumn{2}{|c|}{$\begin{array}{r}\text { Favours } \\
\text { micronutrients }\end{array}$}} & \multirow{2}{*}{$\begin{array}{l}\text { Favours } \\
\text { control }\end{array}$} & \\
\hline & Micronutrients & Control & & & & & \\
\hline \multicolumn{4}{|l|}{ Micronutrients v. placebo } & \multicolumn{2}{|c|}{$\longleftarrow$} & \multicolumn{2}{|l|}{$\longrightarrow$} \\
\hline Christian et al., $2003^{12}$ & $379 / 704$ & $402 / 685$ & $0.92(0.84-1.01)$ & & & & \\
\hline Fawzi et al., $1998^{5}$ & $39 / 410$ & $66 / 390$ & $0.56(0.39-0.81)$ & $\leftarrow$ & & & \\
\hline Overall & $656 / 2520$ & $748 / 2620$ & $0.85(0.71-1.02)$ & & & & \\
\hline \multicolumn{4}{|c|}{ Micronutrients v. iron-folic acid supplementation } & & & & \\
\hline Christian et al., $2003^{12}$ & $379 / 704$ & $327 / 633$ & $1.04(0.94-1.15)$ & & & & \\
\hline Fawzi et al., $2007^{13}$ & $407 / 3827$ & $523 / 3823$ & $0.78(0.69-0.88)$ & & & & \\
\hline Gupta et al., $2007^{15}$ & $23 / 74$ & $31 / 72$ & $0.72(0.47-1.11)$ & & & & \\
\hline \multirow[t]{3}{*}{ Overall } & $1241 / 6529$ & $1372 / 6510$ & $0.89(0.77-1.01)$ & & & & \\
\hline & & & & 0.5 & $0.7 \quad 1$ & $0 \quad 1.5$ & 2.0 \\
\hline & & & & \multicolumn{4}{|c|}{ Relative risk $(95 \% \mathrm{Cl})$} \\
\hline
\end{tabular}

Figure 4: Random-effect meta-analysis of the risk of small-for-gestational-age infants among women given multimicronutrient supplementation versus control (placebo or iron-folic acid supplementation) during pregnancy. Values less than 1.0 indicate decreased risk of outcome with multimicronutrient supplementation. Note: $\mathrm{Cl}=$ confidence interval. 
whereas in other studies it was started as late as in the third trimester. One might have expected stronger evidence of a difference in outcomes with supplementation started before 20 weeks' gestation; however, Shankar and associates ${ }^{21}$ reported a higher efficacy in terms of reduced infant mortality among participants whose supplementation was initiated in their third trimester. Our subgroup analyses did not reveal an advantage with earlier initiation of supplementation; however, the number of studies in each subgroup was small, and there was overlap of the timing of initiation in various studies.

Variation in the composition of micronutrients between the studies was minor except in the study by Fawzi and colleagues, ${ }^{13}$ in which the amount of certain micronutrients was significantly higher than the recommended dietary allowance (Table 2). Our exploratory post-hoc analyses to identify the minimum number of additional nutrients that can be added to an iron-folic acid supplement to identify beneficial effects revealed a combination rather than a particular ingredient that may be essential to observe benefit. However, these results were from a subset of studies that assessed virtually identical compositions of micronutrients. Post-hoc exploration to assess heterogeneity in the treatment effect revealed that treatment effect had no relation with consistent pattern of inclusion or exclusion of vitamin A or zinc, or with particular doses of vitamin A or zinc. We explored these 2 ingredients only because other ingredients of interest were present in similar doses in most formulations of micronu- trients. We assessed publication bias using the funnel plot method; however, this method is exploratory and indirect and may be the result of location of literature, language of publication, citation issues and sample size of the study. No adjustment for publication bias was made in the analyses.

Our findings differ substantially from those reported previously. ${ }^{79}$ Haider and Bhutta ${ }^{7}$ concluded that the evidence was not adequate to replace the current global strategy of iron-folic acid supplementation with multimicronutrient supplementation. Our findings showed that multimicronutrient supplementation was more efficacious than iron-folic acid supplementation in reducing the incidence of low birth weight and in improving birth weight. However, the collection of data differed slightly between the reviews. We included the study by Fawzi and colleagues,$^{13}$ which had been excluded by Haider and Bhutta. ${ }^{7}$ They included a study from Pakistan that we did not include because we could not locate a peer-reviewed article or any other reference for the study. We incorporated new findings from 7 recently completed randomized trials in different countries. We were unable to match some of the numbers for the outcomes included in the Haider and Bhutta review for the study by Christian and colleagues. ${ }^{12}$ We used methods for our meta-analyses that were similar to those used by Haider and Bhutta. As well, we searched clinical trial registries extensively. The variation in multimicronutrient compounds used in the studies was relatively greater in studies conducted before

\begin{tabular}{|c|c|c|c|c|c|c|c|}
\hline \multirow[b]{2}{*}{ Study } & \multicolumn{2}{|c|}{ Micronutrients } & \multicolumn{2}{|r|}{ Control } & \multirow{2}{*}{$\begin{array}{l}\text { Weighted } \\
\text { mean difference, } \\
\text { random }(95 \% \mathrm{Cl})\end{array}$} & \multirow[b]{2}{*}{$\begin{array}{l}\text { Favours } \\
\text { control }\end{array}$} & \multirow[b]{2}{*}{$\begin{array}{l}\text { Favours } \\
\text { micronutrients }\end{array}$} \\
\hline & $n$ & $\begin{array}{l}\text { Weight, g, } \\
\text { mean (SD) }\end{array}$ & $n$ & $\begin{array}{l}\text { Weight, g, } \\
\text { mean (SD) }\end{array}$ & & & \\
\hline \multicolumn{8}{|l|}{ Micronutrients v. placebo } \\
\hline Christian et al., $2003^{12}$ & 705 & 2659 (446) & 635 & $2652(436)$ & $7.00(-40.26$ to 54.26$)$ & & \\
\hline Hininger et al., $2004^{16}$ & 33 & $3300(474)$ & 32 & $3049(460)$ & 251.00 (23.94 to 478.06$)$ & & $\Rightarrow$ \\
\hline Kaestel et al., $2005^{17}$ & 374 & $3097(947)$ & 366 & $3002(956)$ & $95.00(-42.13$ to 232.13$)$ & & > \\
\hline Ramakrishnan et al., $2003^{19}$ & 318 & $2981(381)$ & 315 & $2977(397)$ & $4.00(-56.63$ to 64.63$)$ & & \\
\hline Zeng et al., $2008^{23}$ & 1406 & $3198(438)$ & 1543 & $3158(445)$ & 40.00 (8.11 to 71.89$)$ & & \\
\hline Overall & 2836 & & 2891 & & 31.05 (-5.43 to 67.53$)$ & & \\
\hline \multicolumn{8}{|c|}{ Micronutrients v. iron-folic acid supplementation } \\
\hline Christian et al., $2003^{12}$ & 705 & $2659(446)$ & 685 & $2587(445)$ & 72.00 (25.16 to 118.84$)$ & & \\
\hline Fawzi et al., $2007^{13}$ & 3960 & $3148(530)$ & 3906 & $3083(510)$ & 65.00 (42.02 to 87.98$)$ & & - \\
\hline Gupta et al., $2007^{15}$ & 74 & 2667 (373) & 72 & $2511(419)$ & 156.00 (27.20 to 284.80 ) & & $>$ \\
\hline Osrin et al., $2005^{18}$ & 529 & $2810(453)$ & 523 & $2733(422)$ & 77.00 (24.10 to 129.90$)$ & & \\
\hline Roberfroid et al., $2008^{20}$ & 526 & $2914(450)$ & 526 & $2877(424)$ & $37.00(-15.84$ to 89.84$)$ & & \\
\hline Shankar et al., $2008^{21}$ & 5695 & 3198 (885) & 5406 & $3176(862)$ & $22.00(-10.50$ to 54.50$)$ & & \\
\hline Zagre et al., $2007^{22}$ & 1328 & $3092(190)$ & 1222 & 3025 (205) & 67.00 (51.62 to 82.38$)$ & & $\vdash$ \\
\hline Zeng et al., $2008^{23}$ & 1406 & $3198(438)$ & 1470 & $3174(424)$ & 24.00 (-7.53 to 55.53$)$ & & \\
\hline \multirow[t]{3}{*}{ Overall } & 14223 & & 13810 & & 54.02 (36.22 to 71.83 ) & & \\
\hline & & & & & & -100 & 100 \\
\hline & & & & & & Weighted me & ean difference $(95 \% \mathrm{Cl})$ \\
\hline
\end{tabular}

Figure 5: Random-effect meta-analysis of the effect on birth weight of multimicronutrient supplementation versus control (placebo or iron-folic acid supplementation) during pregnancy. Values greater than zero indicate increased birth weight with multimicronutrient supplementation. Note: $\mathrm{Cl}=$ confidence interval. 
2003 (those included in Haider and Bhutta's review) than in more recent studies included in our review. This was because micronutrient supplementation during pregnancy was highlighted as a research priority and was subsequently investigated with a rather similar approach at the suggestions of UNICEF.

One of the criticisms of any health intervention that is multicomponent in nature is not knowing with certainty which of the components works. It will be difficult to tease out which components of multimicronutrient supplementation were responsible for the improvement in low birth weight that we observed. Therefore, it is not unreasonable to recommend a composite of micronutrients for supplementation because it would be impossible to identify and target individual deficiencies in every pregnant women. The effect of multimicronutrient supplementation on birth weight was relatively smaller than the effect on low birth weight. However, supplementation probably affected infants at a borderline weight around the cutoff of $2500 \mathrm{~g}$.

The number needed to benefit of 33-100 women to prevent 1 low-birth-weight infant is very small from a global perspective. The fourth goal of the United Nations Millennium Declaration (the goals have become known as the Millennium Development Goals) is to reduce child mortality by two-thirds by the year 2015. However, concerns have been raised by the progress made in this direction by several countries. ${ }^{25}$ Low birth weight and related complications are considered the most common cause of mortality globally among infants under the age of 5 years. With the possibility of reducing the incidence of low birth weight by $17 \%$, as shown in our meta-analysis, we believe that providing pregnant women with multimicronutrient supplementation offers the highest possible return for the investment. Current estimates indicate that $15.5 \%$ of about 133 million births worldwide each year are of low-birth-weight infants. We estimate that 75 million births occur in developing countries, where mothers at best receive iron-folic acid supplementation. If all of these women were given multimicronutrient supplementation, our most conservative calculations indicate that about 1.5 million (95\% CI 0.75 million to 2.25 million) births of low-birthweight infants could be averted worldwide every year.

\section{Strengths and limitations}

The strengths of this systematic review include a focused question, an extensive literature search, a large sample of the studied population, inclusion of studies from various parts of the world and the precision of the results. Limitations include clinical heterogeneity in the studies, including the timing and duration of supplementation, the composition of micronutrients and the characteristics of the study populations. All of these features could have an impact on pregnancy outcomes. Further studies are needed to differentiate effects from these perspectives.

\section{Implications for practice and research}

Potential implications for practice include the need to ensure that pregnant women receive multimicronutrient supplementation during the prenatal period. The current strategy recommended by the World Health Organization of providing only iron-folic acid supplementation to pregnant women needs to be challenged in light of the evidence from our review. The education of women of childbearing age on the importance of adequate nutrition for the improvement of pregnancy outcomes should be a priority. Proper advice regarding the use of micronutrients and the elimination of fears and myths regarding their use should be considered.

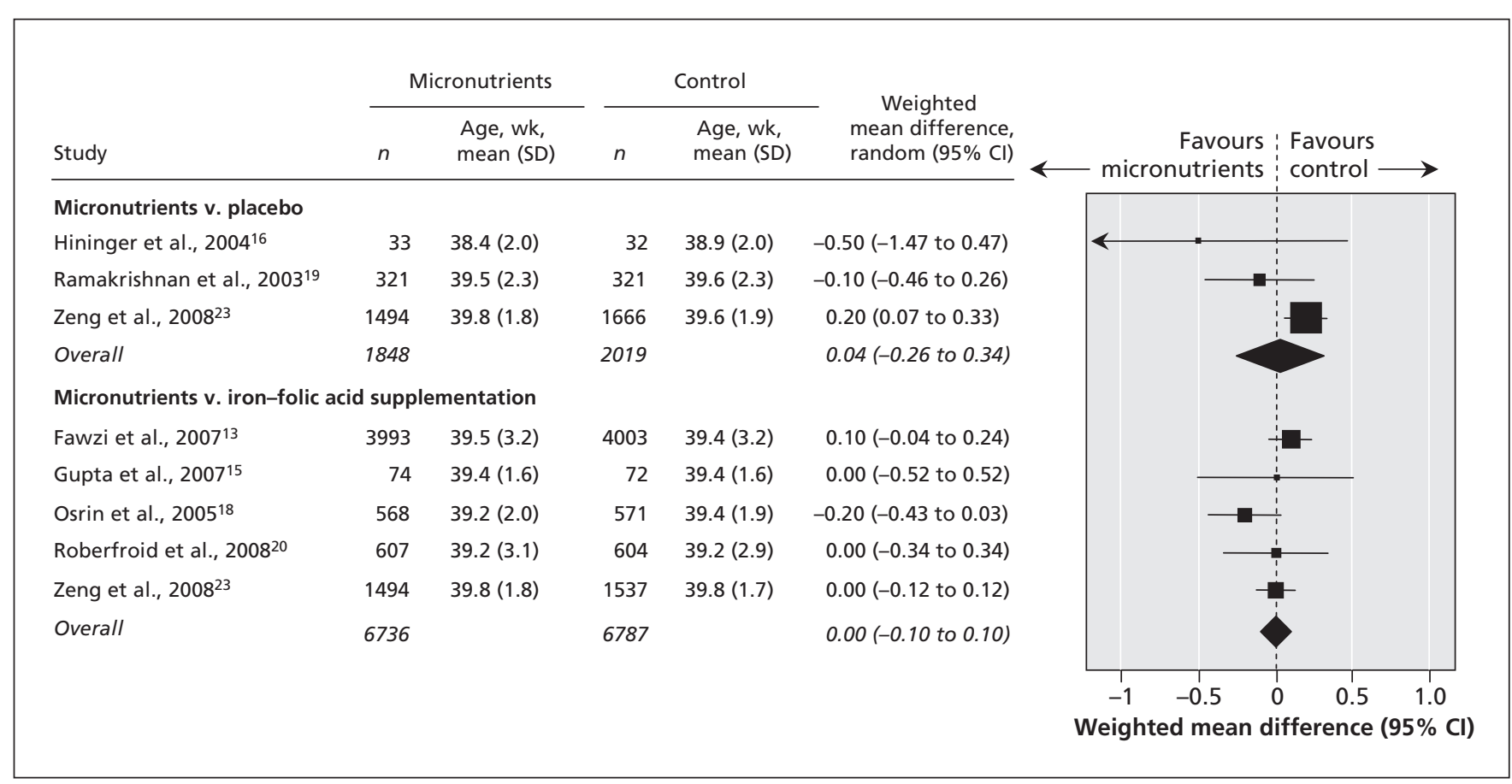

Figure 6: Random-effect meta-analysis of the effect on gestational age of prenatal multimicronutrient supplementation versus control (placebo or iron-folic acid supplementation) during pregnancy. Values less than zero indicate increased gestational age with multimicronutrient supplementation. Note: $\mathrm{Cl}=$ confidence interval. 
Further research is needed to answer certain critical questions. Will a reduction in the risk of low-birth-weight infants associated with multimicronutrient supplementation result in an overall reduction in mortality and related complications among these children? Does multimicronutrient supplementation lead to improved neurodevelopmental outcomes? Do we need different approaches for different populations based on their underlying nutritional status? Will multimicronutrient supplementation at the time of conception improve placentation and prevent pre-eclampsia sufficiently to improve fetal growth? ${ }^{26}$

\section{Conclusion}

We found that prenatal supplementation with multimicronutrients was associated with a significantly reduced risk of low-birth-weight infants and with improved birth weight when compared with iron-folic acid supplementation. We found no effect of multimicronutrient supplementation on the risk of preterm birth or small-for-gestational-age infants. Further research into the use of multimicronutrients at or before conception and its effect on pregnancy outcomes is needed.

\section{This article has been peer reviewed.}

Competing interests: None declared.

Contributors: Prakesh Shah and Arne Ohlsson participated in writing the original grant application and contributed to all drafts of the manuscript. Prakesh Shah was the principal investigator and led the Knowledge Synthesis group. He contributed to analyzing and interpreting the data, wrote the first draft, and is guarantor for this paper. Arne Ohlsson contributed to the conception of the study and protocol, interpreted the data, and revised the first and subsequent drafts of this manuscript. Both authors gave final approval of the manuscript.

Acknowledgement: We thank Elizabeth Uleryk, Chief Librarian at The Hospital for Sick Children, Toronto, Ont., for her contribution in developing the search strategies and running the search on a periodic basis.

Funding: This study was supported by a Knowledge Synthesis/Translation grant (no. KRS 86242) from the Canadian Institute of Health Research (CIHR). CIHR played no role in the analysis or interpretation of the data, the writing of the report or the decision to submit the manuscript for publication.

\section{REFERENCES}

1. Smith CA. Effects of maternal undernutrition upon the newborn infant in Holland (1944-45). J Pediatr 1947;30:229-43.

2. Gopalan C. Multiple micronutrient supplementation in pregnancy. Nutr Rev 2002 60:S2-6.

3. Keen CL, Clegg MS, Hanna LA, et al. The plausibility of micronutrient deficiencies being a significant contributing factor to the occurrence of pregnancy complications. J Nutr 2003;133(Suppl 2):1597S-605S

4. Susser M. Maternal weight gain, infant birth weight, and diet: causal sequences Am J Clin Nutr 1991;53:1384-96.

5. Fawzi WW, Msamanga GI, Urassa W, et al. Vitamins and perinatal outcomes among HIV-negative women in Tanzania. N Engl J Med 2007;356:1423-31.

6. Ladipo OA. Nutrition in pregnancy: mineral and vitamin supplements. Am J Clin Nutr 2000;72(Suppl):280S-90S

7. Haider BA, Bhutta ZA. Multiple-micronutrient supplementation for women during pregnancy [review]. Cochrane Database Syst Rev 2006;(4):CD004905.

8. Composition of a multi-micronutrient supplement to be used in pilot programmes among pregnant women in developing countries. New York (NY): UNICEF; 1999.

9. Lumbiganon P. Multiple-micronutrient supplementation for women during pregnancy [commentary]. Geneva (Switzerland): Reproductive Health Library, World Health Organization; 2007 Available: www.who.int/rhl/pregnancy_childbirth/antenatal_care /nutrition/plcom2/en/index.html (last revised 2007 Aug. 23; accessed 2009 Apr. 29)

10. de Onis M, Villar J, Gülmezoglu M. Nutritional interventions to prevent intrauterine growth retardation: evidence from randomized controlled trials. Eur J Clin Nutr 1998;52(Suppl 1):S83-93.

11. Higgins PT, Green S, editors. Cochrane handbook for systematic reviews of interventions. The Cochrane Collaboration; 2008. Available: www.cochrane-handbook.org (accessed 2008 Jan. 3).

12. Christian P, Khatry SK, Katz J, et al. Effects of alternative maternal micronutrient supplements on low birth weight in rural Nepal: double blind randomised community trial. $B M J$ 2003;326:571.

13. Fawzi WW, Msamanga GI, Spiegelman D, et al. Randomised trial of effects of vitamin supplements on pregnancy outcomes and T cell counts in HIV-1-infected women in Tanzania. Lancet 1998;351:1477-82.

14. Friis H, Gomo E, Nyazema N, et al. Effect of multimicronutrient supplementation on gestational length and birth size: a randomized, placebo-controlled, doubleblind effectiveness trial in Zimbabwe. Am J Clin Nutr 2004;80:178-84.

15. Gupta P, Ray M, Dua T, et al. Multimicronutrient supplementation for undernourished pregnant women and the birth size of their offspring: a double-blind, randomized, placebo-controlled trial. Arch Pediatr Adolesc Med 2007;161:58-64.

16. Hininger I, Favier M, Arnaud J, et al. Effects of a combined micronutrient supplementation on maternal biological status and newborn anthropometrics measurements: a randomized double-blind, placebo-controlled trial in apparently healthy pregnant women. Eur J Clin Nutr 2004;58:52-9.

17. Kaestel P, Michaelsen KF, Aaby P, et al. Effects of prenatal multimicronutrient supplements on birth weight and perinatal mortality: a randomised, controlled trial in Guinea-Bissau. Eur J Clin Nutr 2005;59:1081-9.

18. Osrin D, Vaidya A, Shrestha Y, et al. Effects of antenatal multiple micronutrient supplementation on birthweight and gestational duration in Nepal: double-blind, randomised controlled trial. Lancet 2005;365:916-7.

19. Ramakrishnan U, Gonzalez-Cossio T, Neufeld LM, et al. Multiple micronutrient supplementation during pregnancy does not lead to greater infant birth size than does iron-only supplementation: a randomized controlled trial in a semirural community in Mexico. Am J Clin Nutr 2003;77:720-5.

20. Roberfroid D, Huybregts L, Lanou H, et al. Effects of maternal multiple micronutrient supplementation on fetal growth: a double-blind randomized controlled trial in rural Burkina Faso. Am J Clin Nutr 2008;88:1330-40.

21. Shankar AH, Jahari AB, Sebayang SK, et al. Effect of maternal multiple micronutrient supplementation on fetal loss and infant death in Indonesia: a double-blind cluster-randomised trial. Lancet 2008;371:215-27.

22. Zagre NM, Desplats G, Adou P, et al. Prenatal multiple micronutrient supplementation has greater impact on birthweight than supplementation with iron and folic acid: a cluster-randomized, double-blind, controlled programmatic study in rural Niger. Food Nutr Bull 2007;28:317-27.

23. Zeng L, Dibley MJ, Cheng Y, et al. Impact of micronutrient supplementation during pregnancy on birth weight, duration of gestation, and perinatal mortality in rural western China: double blind cluster randomised controlled trial. BMJ 2008;337:a2001.

24. Ioannidis JP, Trikalinos TA. The appropriateness of asymmetry tests for publication bias in meta-analyses: a large survey. CMAJ 2007;176:1091-6.

25. Mukelabai K. Achieving the Millennium Development Goal to reduce under-five child mortality: a UNICEF perspective. New York (NY): Department of Economic and Social Affairs, Population Division, United Nations; 2008. Available: www.un.org/esa /population/publications/PopAspectsMDG/16_UNICEF1.pdf (accessed 2009 Feb. 17).

26. Catov JM, Bodnar LM, Ness RB, et al. Association of periconceptional multivitamin use and risk of preterm or small-for-gestational-age births. Am J Epidemiol 2007; 166:296-303.

Correspondence to: Dr. Prakesh S. Shah, Associate Professor, Department of Paediatrics, Mount Sinai Hospital, Rm. 775A, 600 University Ave., Toronto ON M5G 1X5; fax 416 586-8745; pshah@mtsinai.on.ca

Members of the Knowledge Synthesis Group on Determinants of Low Birth Weight and Preterm Births: Dr. Prakesh S. Shah, Department of Health Policy, Management and Evaluation, University of Toronto, Toronto, Ont.; Dr. Arne Ohlsson, Departments of Paediatrics, Obstetrics and Gynaecology, and of Health Policy, Management and Evaluation, University of Toronto, Toronto, Ont.; Dr. Vibhuti Shah, Department of Health Policy, Management and Evaluation, University of Toronto, Toronto, Ont.; Dr. Kellie E. Murphy, Department of Obstetrics and Gynecology, University of Toronto, Toronto, Ont.; Dr. Sarah D. McDonald, Departments of Obstetrics and Gynecology and Diagnostic Imaging, McMaster University, Hamilton, Ont.; Dr. Eileen Hutton, Department of Obstetrics and Gynecology, McMaster University, Hamilton, Ont.; Dr. Christine Newburn-Cook, Faculty of Nursing, University of Alberta, Edmonton, Alta.; Dr. Corine Frick, Alberta Perinatal Health Program and Faculty of Nursing, University of Calgary, Calgary, Alta.; Dr. Fran Scott, Dalla Lana School of Public Health, University of Toronto and Toronto Public Health, Toronto, Ont.; Dr. Victoria Allen, Department of Obstetrics and Gynaecology, Dalhousie University, Halifax, NS.; and Dr. Joseph Beyene, Dalla Lana School of Public Health and the Department of Health Policy, Management and Evaluation, University of Toronto, Toronto, Ont. 